\title{
Preoperative imaging for focused parathyroidectomy: making a good strategy even better
}

\section{Carole Guerin ${ }^{1}$, Aoife Lowery ${ }^{1}$, Sophie Gabriel ${ }^{2}$, Frédéric Castinetti ${ }^{3}$, Mélanie Philippon ${ }^{3}$, Josiane Vaillant-Lombard ${ }^{4}$, Anderson Loundou ${ }^{5}$, Jean-François Henry ${ }^{1}$, Frédéric Sebag ${ }^{1}$ and David Taïeb ${ }^{2}$}

Departments of ${ }^{1}$ Endocrine Surgery, ${ }^{2}$ Nuclear Medicine, European Center for Research in Medical Imaging, ${ }^{3}$ Endocrinology and ${ }^{4}$ Radiology, La Timone University Hospital, Aix-Marseille University, 264 Rue Saint-Pierre, 13385 Marseille Cedex 5, France and ${ }^{5}$ Department of Public Health, Aix-Marseille University, Marseille, France
Correspondence should be addressed to D Taïeb

Email

david.taieb@ap-hm.fr

\begin{abstract}
Objective: Surgical treatment for primary hyperparathyroidism (pHPT) has undergone a major paradigm shift during the last decades from bilateral cervicotomy with four-gland neck exploration to image-guided focused approaches. The primary objective of the present study was to compare the performances of parathyroid scintigraphy (PS), parathyroid ultrasonography (US), and the combination of both procedures for guiding a focused approach on the basis of modified interpretation criteria.

Methods: Data from 199 patients operated for apparent sporadic pHPT and evaluated with US and PS using dual-isotope ${ }^{123} \mathrm{I} /{ }^{99 \mathrm{~m}} \mathrm{Tc}$-sestamibi planar pinhole and single-photon emission computed tomography (SPECT) acqusitions were evaluated. Results: A total of 127 patients underwent a focused approach and the remainder had bilateral cervicotomy. In 42 cases, a focused approach was not performed due to the absence of concordant results between US and PS for a single-gland abnormality. Four patients had persistent disease and three had recurrent disease. A localizing preoperative PS had a sensitivity of $93.3 \%$, positive predictive value of $85.8 \%$, negative predictive value of $73.0 \%$, and accuracy of $83.4 \%$ for predicting uniglandular disease. Additional SPECT images accurately localize posterior adenomas that are often missed by US. Compared with PS, US had a lower sensitivity $(P<0.01)$. Our imaging protocol also enabled diagnosis of multiglandular disease in $60.6 \%$.

Conclusions: PS using a highly sensitive dual-tracer subtraction method is the most accurate technique for directing a focused approach. PS could be sufficient for directing a focused approach in the presence of a negative US in two major circumstances: posterior locations due to acquired ectopia that could be missed by US, and previous history of thyroidectomy due to interpretation difficulties.
\end{abstract}

\section{Introduction}

For many years, bilateral cervical exploration with identification of four glands remained the gold standard in parathyroid surgery, and routine preoperative imaging for initial surgery was considered unnecessary and not cost-effective. In 1986, John L Doppman stated 'In my opinion, the only localizing study indicated in a patient with untreated primary hyperparathyroidism (pHPT) is to localize an experienced parathyroid surgeon'. At the same period, some surgeons had developed unilateral parathyroidectomy due to the high frequency of single adenomas (1). The improved performance of radiological and functional investigations has enabled the development of image-guided focused approaches. Bilateral parathyroid exploration is no longer the only option for

Published by Bioscientifica Ltd. 
all patients with pHPT and those with a solitary adenoma on imaging studies can be also candidates for focused approaches.

Parathyroid ultrasonography (US) and scintigraphy (PS) are the imaging modalities most commonly used for selecting candidates for a focused approach. Most institutions use US or PS, either alone or more often both in combination.

Abnormal parathyroid glands are hypoechogenic ovoid structures, whereas normal parathyroid glands are usually invisible on US. US has several advantages over PS: it is inexpensive, widely available, and does not require exposure to ionizing radiation. US also provides information on the thyroid gland. Moreover, US can be performed by surgeons who are aware of the anatomic variants $(2,3)$. This approach might also help to tailor the incision according to the location of the abnormal glands. However, the quality of US is largely dependent on the radiologist's experience and patient's body habitus. Major ectopia (mostly mediastinal) or posterior gland can also be missed by US.

PS has several advantages over US: it can detect major parathyroid ectopia, enables fusion with computed tomography (CT) images, and is more specific. PS might be falsely positive in thyroid nodules, but this drawback can be partly circumvented by subtraction methods (4).

In recent decades, several protocols of parathyroid ${ }^{99 m}$ Tc-sestamibi scintigraphy have been evaluated for parathyroid imaging including single-tracer and dualtracer methods and various acquisition techniques (i.e., planar acquisitions with parallel-hole or pinhole collimators and SPECT or SPECT/CT) (5). For dual-isotope imaging, the use of ${ }^{123} \mathrm{I}$ as a thyroid tracer compared with ${ }^{99} \mathrm{~m}_{\mathrm{TcO}}{ }_{4}^{-}$enables simultaneous acquisition of thyroid and parathyroid images by using a dual-energy window set-up, as first proposed by Hindie et al. (6).

Head-to-head comparison of different protocols has rarely been addressed but dual-tracer methods provide the highest sensitivity $(7,8,9,10,11)$. The use of a pinhole collimator provides a higher sensitivity compared with parallel holes. The combination of a dual-tracer method using ${ }^{123} \mathrm{I} /{ }^{99 \mathrm{~m}} \mathrm{Tc}$-sestamibi and pinhole acquisition is currently the most sensitive protocol for parathyroid imaging $(4,7,11,12)$. Additional SPECT and SPECT/CT acquisitions enable localization of the detected adenomas in all 3D. This provides critical information for choosing the most suitable surgical access to limit surgical exploration and avoid recurrent laryngeal nerve injury $(13,14)$.

The reported accuracy of PS and US does not represent the true efficacy of these techniques for directing focused parathyroidectomy, because reported accuracy is usually performed on the basis of head-to-head comparison between imaging findings and abnormal glands (15). The primary objective of this study was to compare the performances of neck US, PS and the combination of both procedures for guiding a focused approach. To this end, the efficacy of imaging studies was based on modified interpretation criteria to determine specifically whether they could influence the surgeon's choice of a focused vs a conventional approach to parathyroidectomy. For example, a study showing only a single parathyroid lesion in a patient with double hyperfunctioning adenomas has been interpreted as false-positive (FP) result because it should lead to an inappropriate focused surgical approach with a possible subsequent surgical conversion. By contrast, a negative study in the presence of multiglandular disease (MGD) should lead to appropriate bilateral open-surgery and should be interpreted as a true-negative (TN) study for single-gland disease.

\section{Patients and methods}

Between April 2011 and April 2013, 445 patients with biochemical evidence of apparently sporadic pHPT (absence of another endocrine tumor, negative family history) underwent parathyroidectomy at the Department of Endocrine Surgery of La Timone University Hospital. Preoperative imaging included neck ultrasonography (US) and PS. From April 2012 to the study conclusion, patients who presented with imaging investigations performed at another institution systematically had preoperative imaging repeated in our institution. Only those who were evaluated by US and dual-isotope $\left({ }^{123} \mathrm{I} /{ }^{99 \mathrm{~m}} \mathrm{Tc}\right.$-sestamibi) PS protocol in our institution were included. Thus, 199 patients treated surgically constitute our study population.

According to the results of the preoperative imaging workup, patients underwent surgery via a focused approach in case of positive imaging findings suggestive of single-gland disease (most often on both US and PS) and absence of thyroid disease that warranted a transverse cervicotomy approach. A focused approach included both mini-invasive cervicotomy (anterior parathyroid) and lateral endoscopic approach (posterior parathyroid). In patients who underwent a focused approach, intraoperative parathyroid hormone (IOPTH) values after excision were used as a guide for when to stop surgery $(50 \%$ or more drop and normalization of parathyroid hormone (PTH) level) or convert to bilateral exploration. All patients had fasting calcium and PTH 
levels measured on day one by spectrophotometry using the chromophore NM-BAPTA for calcium and with an electrochemiluminescence immunoassay for intact PTH (ECLIA, Roche-Diagnostics, Mannheim, Germany). Medium-term follow-up data were available for 141 patients with calcium and PTH levels assessed at least 6 months after surgery.

In keeping with Local Institutional Guidelines, all patients gave informed consent for the use of anonymous personal data extracted from their medical records for scientific or epidemiological purposes.

\section{Neck US}

Neck ultrasound scanning (neck US) was performed in all patients. Neck US was performed with the neck in extension, by a single radiologist with 30-years experience (J V) using a Philips IU22 with a 12-MHz linear transducer. The area examined by neck US extended from the angle of the mandible to the superior part of the anterior mediastinum (examined by inclining the transducer toward the retrosternal region).

\section{Parathyroid scintigraphy}

All patients underwent dual-isotope $\left({ }^{123} \mathrm{I} /{ }^{99 \mathrm{~m}} \mathrm{Tc}\right.$-sestamibi) static planar pinhole imaging (pinhole collimators) followed by ${ }^{99 m}$ Tc-sestamibi SPECT (parallel-hole collimators) on the same day. The same camera (Siemens ECAM, Siemens Medical Systems, Erlangen, Germany) was used for both image acquisitions. The steps of the protocol were as follows. First, the patients received $12 \mathrm{MBq}$ of ${ }^{123} \mathrm{I}$. Two hours later, $740 \mathrm{MBq}$ of ${ }^{99 \mathrm{~m}}$ Tc-sestamibi was injected. Pinhole acquisition was started $3 \mathrm{~min}$ after ${ }^{99 \mathrm{~m}}$ Tc-sestamibi injection; static anterior cervical views were obtained for $20 \mathrm{~min}$ with two photopeaks of 7\% energy windows, centered, respectively, over the $140 \mathrm{keV}\left({ }^{99 \mathrm{~m}} \mathrm{Tc}\right)$ and $164 \mathrm{keV}\left({ }^{123} \mathrm{I}\right)$ photopeaks $(128 \times 128$ matrix, zoom of 2.67). Interactive software was used for image normalization and subtraction. The tomographic study was then started with parallel-hole collimators (at 30-45 min after sestamibi injection), with the following parameters: $128 \times 128$ matrix, zoom of $1.45,45 \mathrm{~s}$ per projection at each of 32 angular steps, and sampling of a $360^{\circ}$ orbit. The photopeak was centered over $140 \mathrm{keV}$ only, and iterative reconstruction was performed with a Gaussian filter (full width at half maximum, $10 \mathrm{~mm}$ ). The neck and the mediastinum (from the angle of the mandible to the heart) were included in the field of view.

\section{Image interpretation}

On neck US images, a positive finding was defined by an ovoid homogeneous hypoechogenic gland, with peripheral vascularization.

On planar pinhole images, a positive finding was defined as residual ${ }^{99 \mathrm{~m}}$ Tc activity after normalization and subtraction. On SPECT, a positive finding was defined as an extrathyroidal focal uptake (posterior or inferior to the thyroid lobe, mediastinal). The lesions were classified as superior ( $\mathrm{P} 4$ derived) if they were posterior and located within the superior two-thirds of the thyroid lobe. They were classified as P3 if they were anterior and developed at the tip of the inferior pole of the thyroid lobe or along the thyrothymic tract. Other locations included adenomas that were described as anterior or posterior (14).

\section{Disease status}

Cure was defined irrespective of surgical strategy by normalization of serum calcium at least 6 months after parathyroidectomy. In the absence of follow-up data, patients were classified on the basis of their latest followup for serum calcium assessment.

Uniglandular disease (UGD) was defined when only a single abnormal gland was removed and the patient was cured. When more than one gland was involved histologically (adenoma or hyperplasia), the patient was considered to have MGD, including cases with multiple adenomas and multiple gland parathyroid hyperplasia. Patients not cured after excision of a single lesion were also considered to have MGD.

\section{Statistical analyses}

The efficacy of imaging studies was based on modified interpretation criteria to determine specifically whether they could adequately guide the surgeon's choice of a neck-focused approach vs a conventional approach to parathyroidectomy. We wanted to be clinically realistic, because when performing a focused approach, the priority of the surgeon is to know that the procedure will be curative. In the case of a single abnormality, concordance was defined as strictly the same localization on US, PS, and surgical findings. By contrast, a negative study in the presence of MGD should lead to appropriate bilateral open-surgery and should be interpretated as a TN study for single-gland disease. 
According to the results of neck US and PS and of our criteria for disease status, all patients were classified as follows:

True-positive (TP) result: a single cervical abnormality on US or PS or both (concordant abnormality)* and a single concordant UGD.

FP result: a single cervical abnormality on US or PS or both* and MGD or UGD found in a different location. TN result: absence or two or more abnormalities on US or PS and MGD or major ectopia; or absence of concordance for a cervical single abnormality on US+ PS* and MGD or major ectopia.

False-negative (FN) result: absence or two or more abnormalities on US or PS and UGD in the neck; or absence of concordance for a cervical single abnormality on US +PS* and UGD in the neck.

According to our criteria, positive predictive value (PPV) was the probability that the imaging investigation correctly predicted UGD and the negative predictive value (NPV) was the probability that the imaging investigation correctly excluded UGD.

These results were used to calculate the conventional screening assessment and their CIs are calculated: sensitivity (Se), PPV, NPV, and accuracy (Acc) of US, PS, and the combination of both. Specificity was not calculated because the analysis was not performed at a lesion-level.

Between-group comparisons were performed using $\chi^{2}$ or Fisher's exact test for qualitative variables and Student's $t$-test or Mann-Whitney $U$ test for continuous characteristics (expressed as mean \pm s.D. or median with interquartile range (IQR) if appropriate). The extended McNemar test was used to compare sensitivities of the different imaging modalities.

The $P$ values of $\sim 0.05$ were taken to be statistically significant. All statistical analyses were performed using SPSS 17.0 Software.

\section{Results}

\section{Patients and parathyroid disease}

In the study, 163 of the 199 patients were female. The median age was 64 years ( $\mathrm{IQR}=54-73$ years). Preoperative median serum calcium and PTH levels were $2.68 \mathrm{mmol} / 1$ $(\mathrm{IQR}=2.62-2.78) \quad$ and $12.7 \mathrm{pmol} / \mathrm{l} \quad(\mathrm{IQR}=9.7-17.2)$ respectively. A total of 163 UGD (162 in the neck and one major ectopia in the aorto-pulmonary window) and 36 MGD were observed. No significant difference in median age $(P=0.07)$, preoperative serum calcium
$(P=0.2)$ or PTH $(P=0.51)$ was observed in patients with UGD compared with those with MGD.

Disease status was defined on the basis of extended follow-up data in 141 cases ( $>6$ months) and earlier post-operative evaluations in the remaining 58 patients. The median follow-up was 12 months $\mathrm{IQR}=8$ days -17 months).

A total of 192 achieved remission criteria. Seven patients were classified as persistent/recurrent pHPT: one patient underwent conversion based on a failure of IOPTH drop-off and had a MGD, one was not converted to a bilateral exploration despite failure of IOPTH drop-off due to the presence of a recurrent laryngeal nerve injury. The remaining five patients (two persistent/three recurrent) had a 50\% decrease in IOPTH and normalization of PTH and had a serum calcium above the URL but below $2.60 \mathrm{mmol} / 1$ after 6-month follow-up.

\section{Imaging findings and surgical approaches}

US localized 194 parathyroid imaging abnormalities and PS localized 210 parathyroid imaging abnormalities. A single abnormality was found in 157 patients with neck US and in 162 patients with PS. In 124 cases, US and PS showed a single cervical abnormality. In 114 cases, US and PS found a concordant single-cervical abnormality, six had nonconcordant single-cervical abnormality (same side but not the same gland). More than one abnormality was found on US and PS in 18 and 23 patients respectively. No abnormalities were found by US in 24 patients (including the patient with major ectopia) and by PS in 13 patients. In five cases, both US and PS were negative.

A total of 127 patients who underwent a focused approach had a single concordant abnormality on US and PS in 112 cases, a single positive finding on PS in 13 cases and a single abnormality on US in the latter case.

Table 1 Reasons for not performing focused approach in patients with neck abnormalities.

\begin{tabular}{|c|c|c|}
\hline Cause & $\begin{array}{l}\text { No. of } \\
\text { patients }\end{array}$ & $\begin{array}{c}\text { Percentage of total } \\
\text { conventional } \\
\text { operations }\end{array}$ \\
\hline Thyroid resection $^{a}$ & 23 & 31.9 \\
\hline $\begin{array}{l}\text { Cervical reoperations } \\
\text { (not for HPT) }\end{array}$ & 3 & 4.2 \\
\hline Other reasons & 2 & 2.7 \\
\hline Localization problems & 43 & 59.8 \\
\hline Conversion & 1 & 1.4 \\
\hline Total & 72 & 100 \\
\hline
\end{tabular}


Table 2 Performances of US, PS, and US+PS for predicting UGD.

\begin{tabular}{|c|c|c|c|c|}
\hline & TP & FP & $\mathbf{T N}$ & $\mathbf{F N}$ \\
\hline US $(n=199)$ & 124 & 34 & 18 & 23 \\
\hline PS & 139 & 23 & 27 & 10 \\
\hline PS + US & 112 & 12 & 28 & 45 \\
\hline
\end{tabular}

\begin{tabular}{c}
\hline Se $(95 \% \mathrm{Cls})$ \\
\hline $84.5(77.6-89.3)$ \\
$93.3(88.1-96.3)$ \\
$71.3(63.8-77.8)$ \\
\hline
\end{tabular}

\begin{tabular}{c}
\hline PPV $(95 \% \mathrm{Cls})$ \\
\hline $78.5(71.4-84.2)$ \\
$85.8(79.6-90.4)$ \\
$90.3(83.8-94.4)$ \\
\hline
\end{tabular}

Accuracy (95\% Cls)

$71.4(64.7-77.2)$

$83.4(77.6-87.9)$

$71.1(64.4-76.9)$
One patient underwent video thoracoscopy for resection of an adenoma located in the aorto-pulmonary window and accurately detected by PS.

The remaining 72 patients underwent bilateral cervicotomy. The reasons for not performing focused approach are described in Table 1. In 43 cases, focused approach was not performed due to the absence of concordant results for a single-gland abnormality. Thirty patients had concomitant thyroidectomy. In 23 cases, bilateral cervicotomy was performed because of concomitant total thyroidectomy. The remaining seven cases had a thyroid lobectomy without exploration of the contralateral side and were therefore considered as focused approaches.

\section{Comparison between PS, US, and PS + US}

A localizing preoperative PS had a sensitivity of $93.3 \%$ (95\% CI: 88.1-96.3), PPV of 85.8\% (95\% CI: 79.6-90.4), and NPV of $73.0 \%$ (95\% CI: 57.0-84.6) for directing a focused approach (Table 2). Compared with PS, US had an $8.9 \%$ lower sensitivity $(P<0.01)$ (negative US in 19 cases with UGD and multiple abnormalities in four cases with UGD). In 16/19 cases, US was negative but PS found a single abnormality in the neck concordant with surgical findings: $13 / 16$ were posterior glands and 3/16 were anterior glands on SPECT images. In 2/19 cases, patients had a previous history of thyroidectomy. Examples of FN results of US are illustrated in Figs 1 and 2. The last patient was operated via a thoracoscopy approach.

In patients with MGD $(n=36), 12$ had more than one abnormality on PS and US and two had both negative imaging. A single abnormality was found by US and PS respectively in 18 and ten cases. In 5/6 cases, both US and PS were concordant on one abnormality. 4/5 were considered uncured at the end of the study. The remaining cases had discordant imaging finding (Table 3).

Statistically, the association of US + PS had higher PPV in comparison with US alone but not with PS alone (Table 2).

\section{Discussion}

Surgical treatment for pHPT has undergone a major paradigm shift during the last decades. It has shifted from the $\mathrm{BC}$ with four-gland neck exploration to a focused approach. In this study, $64 \%$ of patients have benefited from a focused approach (including a video thoracoscopy in one case). It is remarkable that the absence of concordance between both studies was the main reason for not performing a focused approach. The present study shows that PS using ${ }^{123} \mathrm{I} /{ }^{99 \mathrm{~m}} \mathrm{Tc}$-sestamibi pinhole planar method and SPECT appears to be the most accurate strategy for directing a focused approach. One of the originalities of the study was to use modified interpretation criteria for PS and US in order to determine specifically whether they could influence the surgeon's

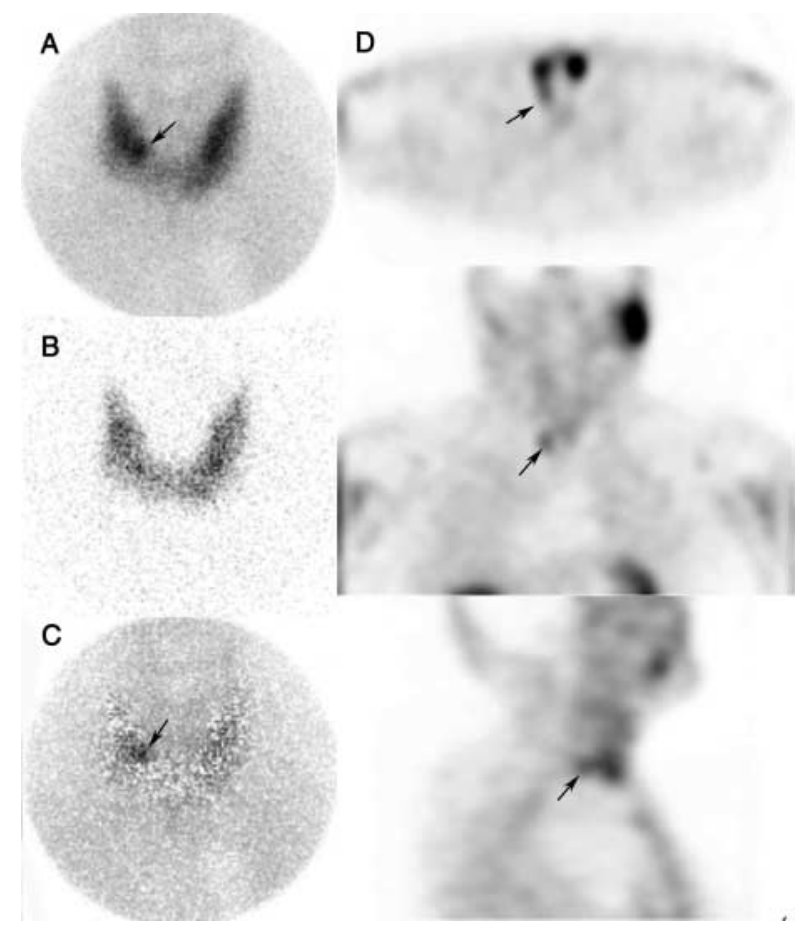

\section{Figure 1}

Planar pinhole (A: ${ }^{99 m} \mathrm{Tc}$-sestamibi, B: ${ }^{123} \mathrm{I}, \mathrm{C}$ : subtraction images) and SPECT images (D) of parathyroid adenomas. Typical small adenoma located in the inner part of the right thyroid lobe above the isthmus (arrows). Taken together with the posterior extension of the adenoma on SPECT images, this feature is highly suggestive of P4 origin (arrows). The lesion was missed by US. Final diagnosis: right P4 adenoma. 

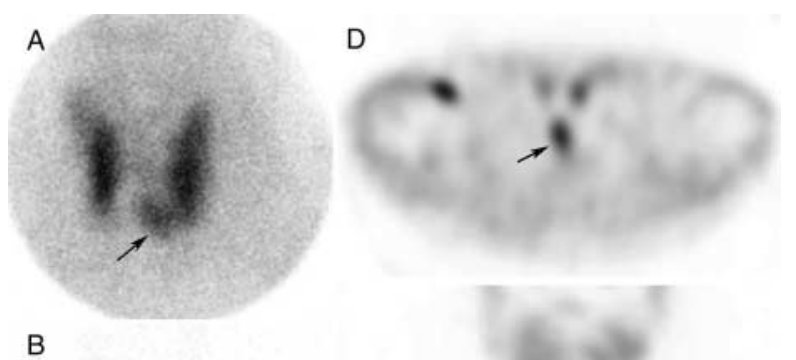

\section{Figure 2}

Planar pinhole (A: ${ }^{99 \mathrm{~m}} \mathrm{Tc}$-sestamibi, B: ${ }^{123} \mathrm{I}, \mathrm{C}$ : subtraction images) and SPECT images (D) of parathyroid adenomas. Typical adenoma located in the inner part of the inferior pole of the left thyroid lobe (arrows). The posterior extension of the adenoma on SPECT images is highly suggestive of P4 origin (arrows). The lesion was missed by US. Final diagnosis: right $\mathrm{P} 4$ adenoma.

choice of a focused vs a conventional approach to parathyroidectomy.

PS and US are the preferred approaches reported by endocrine surgeons in the US for selecting candidates for a focused approach (16). Recently, Kunstman et al. (17) have suggested that a single-positive or suggestive imaging modality (PS or US) may be a sufficient indication for focused approaches. Our results show that dualradiopharmaceutical ${ }^{123} \mathrm{I} /{ }^{99 \mathrm{~m}} \mathrm{Tc}$-sestamibi subtraction pinhole imaging followed by SPECT acquisition is more accurate than US for directing a focused approach $(4,7,11,12)$. Our protocol avoids motion artifacts because it is based on a simultaneous acquisition of the two photopeaks $(4,6,8,18)$. However, our protocol requires long acquisition times ( $20 \mathrm{~min}$ for pinhole acquisition) to improve its sensitivity, but does not require delayed images as those for dual-phase protocols. Our protocol is also more expensive due to the cost of ${ }^{123}$ I. A preoperative
PS and US that localized a single parathyroid adenoma had a PPV of 85.8 and $78.5 \%$ respectively. The use of concordant imaging findings as a rule for directing a focused approach has a high PPV (90.3\%) (95\% CI: 83.8-94.4) but reduces the number of patients eligible for this approach. However, we found 23 FP results for PS, including MGD (11/23) and a single-gland disease with discordant location (12/23) (anterior vs posterior).

As in previous series, adenomas missed by US were predominantly posterior in our study (19). Thus, we agree that additional SPECT images are required for visualizing these adenomas in all 3D. This information is also important for choosing the most suitable surgical access such as, for example, a central mini-open approach for anterior lesions or a lateral endoscopic approach for posterior adenomas. With the improvement of PS protocols, it is also possible to accurately detect adenomas in patients with a previous history of thyroidectomy that could be missed by US (often misdiagnosed as thyroid remnants).

In our opinion, discordant findings between US/PS still require a four-gland identification approach in order to limit the rate of persistent disease. However, PS could be sufficient for directing a focused approach in cases where the US is negative due to posterior or ectopic location of the parathyroid adenoma, or in cases of interpretation difficulty due to previous thyroidectomy.

In cases with concordant imaging findings, US provides additional anatomic information on the thyroid gland (volume, nodules) and parathyroid tumors (volume, shape) that could potentially influence surgical approach. A total of seven patients had persistent $(n=4)$ or recurrent pHPT $(n=3)$. IOPTH failed to predict persistent/recurrent disease in 5/7 cases. However, diagnosis of recurrence remains questionable because serum calcium levels were slightly above the normal range but remained $<2.60 \mathrm{mmol} / \mathrm{l}$.

In our series, the incidence of MGD was $18 \%$, which was within the range of $3-24 \%$ reported in the

Table 3 Imaging findings in patients with MGD $(n=36)$.

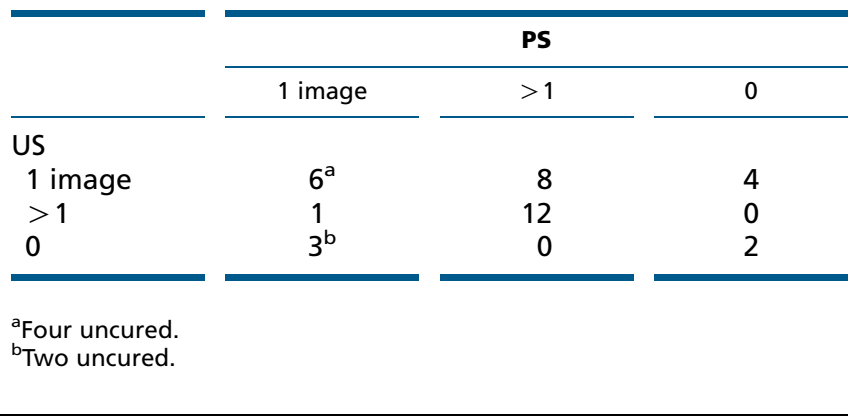


literature (20). The rate of MGD in cases of negative PS has been reported as $\sim 25 \%(21,22,23)$. Therefore, negative localization with sestamibi infers a highly selected patient population with small parathyroid adenomas and/or MGD. It has been shown that the cure rate was lower in this subgroup of patients $(21,22)$. It is currently accepted that besides some cellular-molecular specificities, one possible explanation for decreased detection of MGD (often hyperplasic) is related to their smaller volume compared with adenomas. In our series, the rate of MGD in cases of negative PS was 6/13 (46.2\%). Our imaging protocol enabled the visualization of multiple foci in 20/33 cases of MGD (60.6\%). Taken together, the NPV of PS for UGD was 73\%, which is higher than US alone and the combination of US and PS. Our data are in agreement with early series that have found high sensitivity of dualisotope scintigraphy with pinhole imaging in detection of MGD defined on the basis of parathyroid four-gland identification $(18,24)$. Head-to-head comparison between ${ }^{123} \mathrm{I} /{ }^{99 \mathrm{~m}} \mathrm{Tc}$-sestamibi PS and the recently introduced ${ }^{18} \mathrm{~F}$-fluorocholine PET/CT in a large series of pHPT, including medico-economic aspects, will be of particular interest $(25,26)$.

In conclusion, ${ }^{123} \mathrm{I} /{ }^{99 \mathrm{~m}} \mathrm{Tc}$-sestamibi using a pinhole collimator is a reliable method for selecting the optimal candidates for a focused approach, and may be sufficient when US is negative in the presence of posterior adenomas, major ectopia or in cases of previous cervical surgery. This method also facilitates the detection of MGD in the majority of cases.

\section{Declaration of interest}

The authors declare that there is no conflict of interest that could be perceived as prejudicing the impartiality of the research reported.

\section{Funding}

This research did not receive any specific grant from any funding agency in the public, commercial or not-for-profit sector.

\section{References}

1 Tibblin S, Bondeson AG \& Ljungberg O. Unilateral parathyroidectomy in hyperparathyroidism due to single adenoma. Annals of Surgery 1982 195 245-252. (doi:10.1097/00000658-198203000-00001)

2 Stephen AE, Milas M, Garner CN, Wagner KE \& Siperstein AE. Use of surgeon-performed office ultrasound and parathyroid fine needle aspiration for complex parathyroid localization. Surgery 2005138 1143-1150; discussion 1150-1151. (doi:10.1016/j.surg.2005.08.030)

3 Deutmeyer C, Weingarten M, Doyle M \& Carneiro-Pla D. Case series of targeted parathyroidectomy with surgeon-performed ultrasonography as the only preoperative imaging study. Surgery 2011150 1153-1160. (doi:10.1016/j.surg.2011.09.041)

4 Hindie E, Ugur O, Fuster D, O'Doherty M, Grassetto G, Urena P, Kettle A, Gulec SA, Pons F \& Rubello D. EANM parathyroid guidelines. European Journal of Nuclear Medicine and Molecular Imaging 200936 1201-1216. (doi:10.1007/s00259-009-1131-z)

5 Taieb D, Hindie E, Grassetto G, Colletti PM \& Rubello D. Parathyroid scintigraphy: when, how, and why? A concise systematic review Clinical Nuclear Medicine 201237 568-574. (doi:10.1097/RLU. Ob013e318251e408)

6 Hindie E, Melliere D, Perlemuter L, Jeanguillaume C \& Galle P. Primary hyperparathyroidism: higher success rate of first surgery after preoperative Tc-99m sestamibi-I-123 subtraction scanning. Radiology 1997204 221-228. (doi:10.1148/radiology.204.1.9205251)

7 Caveny SA, Klingensmith WC III, Martin WE, Sage-El A, McIntyre RC Jr Raeburn C \& Wolfe P. Parathyroid imaging: the importance of dualradiopharmaceutical simultaneous acquisition with ${ }^{99 \mathrm{~m}} \mathrm{Tc}$-sestamibi and ${ }^{123}$ I. Journal of Nuclear Medicine Technology 201240 104-110. (doi:10.2967/jnmt.111.098400)

8 Hindie E, Melliere D, Jeanguillaume C, Perlemuter L, Chehade F \& Galle P. Parathyroid imaging using simultaneous double-window recording of technetium-99m-sestamibi and iodine-123. Journal of Nuclear Medicine 199839 1100-1105.

9 Leslie WD, Dupont JO, Bybel B \& Riese KT. Parathyroid ${ }^{99 \mathrm{~m}}$ Tc-sestamibi scintigraphy: dual-tracer subtraction is superior to double-phase washout. European Journal of Nuclear Medicine and Molecular Imaging 200229 1566-1570. (doi:10.1007/s00259-002-0944-9)

10 Neumann DR, Esselstyn CB Jr, Go RT, Wong CO, Rice TW \& Obuchowski NA. Comparison of double-phase ${ }^{99 \mathrm{~m}} \mathrm{Tc}$-sestamibi with ${ }^{123} \mathrm{I}_{-}{ }^{99 \mathrm{~m}} \mathrm{Tc}$-sestamibi subtraction SPECT in hyperparathyroidism. AJR. American Journal of Roentgenology 1997169 1671-1674. (doi:10.2214/ajr.169.6.9393188)

11 Tunninen V, Varjo P, Schildt J, Ahonen A, Kauppinen T, Lisinen I, Holm A, Eskola H \& Seppanen M. Comparison of five parathyroid scintigraphic protocols. International Journal of Molecular Imaging 2013 2013 921260. (doi:10.1155/2013/921260)

12 Klingensmith WC III, Koo PJ, Summerlin A, Fehrenbach BW, Karki R, Shulman BC, Raeburn CD \& McIntyre RC Jr. Parathyroid imaging: the importance of pinhole collimation with both single- and dual-tracer acquisition. Journal of Nuclear Medicine Technology 201341 99-104. (doi:10.2967/jnmt.112.118208)

13 Hassler S, Ben-Sellem D, Hubele F, Constantinesco A \& Goetz C. Dual-isotope ${ }^{99 \mathrm{~m}} \mathrm{Tc}-\mathrm{MIBI} /{ }^{123} \mathrm{I}$ parathyroid scintigraphy in primary hyperparathyroidism: comparison of subtraction SPECT/CT and pinhole planar scan. Clinical Nuclear Medicine 201439 32-36. (doi:10.1097/RLU.0000000000000272)

14 Taieb D, Hassad R, Sebag F, Colavolpe C, Guedj E, Hindie E, Henry JF \& Mundler $\mathrm{O}$. Tomoscintigraphy improves the determination of the embryologic origin of parathyroid adenomas, especially in apparently inferior glands: imaging features and surgical implications. Journal of Nuclear Medicine Technology 200735 135-139. (doi:10.2967/jnmt.107. 039743)

15 Kim CK, Kim S, Krynyckyi BR, Machac J \& Inabnet WB. The efficacy of sestamibi parathyroid scintigraphy for directing surgical approaches based on modified interpretation criteria. Clinical Nuclear Medicine 2002 27 246-248. (doi:10.1097/00003072-200204000-00002)

16 Greene AB, Butler RS, McIntyre S, Barbosa GF, Mitchell J, Berber E, Siperstein A \& Milas M. National trends in parathyroid surgery from 1998 to 2008: a decade of change. Journal of the American College of Surgeons 2009209 332-343. (doi:10.1016/j.jamcollsurg.2009.05.029)

17 Kunstman JW, Kirsch JD, Mahajan A \& Udelsman R. Clinical review: parathyroid localization and implications for clinical management. Journal of Clinical Endocrinology and Metabolism 201398 902-912. (doi:10.1210/jc.2012-3168)

18 Hindie E, Melliere D, Jeanguillaume C, Urena P, deLabriolle-Vaylet C \& Perlemuter L. Unilateral surgery for primary hyperparathyroidism on 
the basis of technetium Tc $99 \mathrm{~m}$ sestamibi and iodine 123 subtraction scanning. Archives of Surgery 2000135 1461-1468. (doi:10.1001/ archsurg.135.12.1461)

19 Harari A, Mitmaker E, Grogan RH, Lee J, Shen W, Gosnell J, Clark O \& Duh QY. Primary hyperparathyroidism patients with positive preoperative sestamibi scan and negative ultrasound are more likely to have posteriorly located upper gland adenomas (PLUGs). Annals of Surgical Oncology 201118 1717-1722. (doi:10.1245/s10434-010-1493-2)

20 Chiu B, Sturgeon C \& Angelos P. What is the link between nonlocalizing sestamibi scans, multigland disease, and persistent hypercalcemia? A study of 401 consecutive patients undergoing parathyroidectomy. Surgery 2006140 418-422. (doi:10.1016/j.surg. 2006.03.021)

21 Bergenfelz AO, Wallin G, Jansson S, Eriksson H, Martensson H, Christiansen P \& Reihner E. Results of surgery for sporadic primary hyperparathyroidism in patients with preoperatively negative sestamibi scintigraphy and ultrasound. Langenbeck's Archives of Surgery 2011 396 83-90. (doi:10.1007/s00423-010-0724-0)

22 Dy BM, Richards ML, Vazquez BJ, Thompson GB, Farley DR \& Grant CS. Primary hyperparathyroidism and negative Tc99 sestamibi imaging: to operate or not? Annals of Surgical Oncology 201219 2272-2278. (doi:10.1245/s10434-012-2325-3)
23 Sebag F, Hubbard JG, Maweja S, Misso C, Tardivet L \& Henry JF. Negative preoperative localization studies are highly predictive of multiglandular disease in sporadic primary hyperparathyroidism. Surgery 2003134 1038-1041; discussion 1041-1042. (doi:10.1016/ j.surg.2003.07.021)

24 Johnston LB, Carroll MJ, Britton KE, Lowe DG, Shand W, Besser GM \& Grossman AB. The accuracy of parathyroid gland localization in primary hyperparathyroidism using sestamibi radionuclide imaging. Journal of Clinical Endocrinology and Metabolism $1996 \mathbf{8 1}$ 346-352.

25 Lezaic L, Rep S, Sever MJ, Kocjan T, Hocevar M \& Fettich J. (1)(8)F-Fluorocholine PET/CT for localization of hyperfunctioning parathyroid tissue in primary hyperparathyroidism: a pilot study. European Journal of Nuclear Medicine and Molecular Imaging 201441 2083-2089. (doi:10.1007/s00259-014-2837-0)

26 Michaud L, Burgess A, Huchet V, Lefevre M, Tassart M, Ohnona J, Kerrou K, Balogova S, Talbot JN \& Perie S. Is ${ }^{18}$ F-fluorocholinepositron emission tomography/computerized tomography a new imaging tool for detecting hyperfunctioning parathyroid glands in primary or secondary hyperparathyroidism? Journal of Clinical Endocrinology and Metabolism 201499 4531-4536. (doi:10.1210/ jc.2014-2821)

Received 10 November 2014

Revised version received 30 January 2015

Accepted 30 January 2015 